\title{
MODE I FAILURE OF LAMINATED POLYMERIC COMPOSITES
}

\author{
SEUNG J. SONG and ANTHONY M. WAAS \\ Department of Aerospace Engineering, College of Engineering, University of Michigan, Ann Arbor, \\ MI 48109-2118, U.S.A.
}

\begin{abstract}
A mechanical model for the prediction of mode I deiamination failure of a laminated double cantilever beam (DCB) type specimen is presented. The volume of material ahead of the crack tip that experiences high stresses due to the presence of the crack tip was replaced by a nonlincar clastic spring foundation. The volume undergoing global deformation due to the external loading was replaced by a beam. The spring foundation was characterized by a covalent interatomic force law as a constitutive law and a non-uniform strain distribution throughout the spring length. Experimental data of fracture toughness for PEEK adhesive joints were used to partially characterize the spring foundation. Experimental results from mode I fracture tests performed to verify the current model are presented. The current model matched the experimental results closely for PEEK and BP907 adhesive joints for a wide range of adhesive layer thickness. It also reproduced load vs displacement curves of E7TI/G40 and E7/9/1M7 composite specimens very closely. The work presented contributes a new fracture model for prediction of delamination of laminated composite structures.
\end{abstract}

\section{INTRODUCTION}

DELAMINATION failure in laminated composites is a subject that has been receiving a considerable amount of attention as evidenced by the large number of papers devoted to this topic. The results of a combined experimental and analytical study are reported herein, where a novel and systematic way to model mode I delamination failure in continuous fiber unidirectionally laminated composites is considered.

Fracture mechanics concepts based on a critical energy release rate have not lent themselves readily in applications to fracture problems of non-homogeneous structures such as layered materials. The complexity that is present in such a structure inhibits its application from being an easy task. The application even becomes impossible for a certain combination of materials in layered structures [1]. Besides, the meaning of a critical energy release rate and its proper interpretation is not well understood for this type of structure. Chai $[2,3]$ experimentally showed that the fracture toughness, or so called critical energy release rate for a given material is not a true material constant but varies considerably depending on the thickness of the material. Additionally, two major difficulties associated with LEFM concepts arise in addressing problems of crack growth. First, for crack growth in any material (layered or otherwise), one has to adopt a "fracture criterion (or law)" to define the onset of crack growth. In an energetic setting, this law consists of an equation composed of the different components of energy release rates in some functional description, quite similar to yield criteria encountered in the theory of plasticity. Thus, for crack propagation, one has to compute the different components of strain energy release rate or in some cases the total strain energy release rate, depending on the growth law that is chosen. Once the geometry of the cracked body and the direction of crack growth are known, computing the components of strain energy release rate (or the total) for a homogeneous continuum is well understood [1]. However, in a strict 2-D setting, such as a crack front with curvature, it is not at all clear how one can compute strain energy release rates at every point along the crack boundary. It appears that the notion of a strain energy release rate, which, by definition is dependent on "per unit of future cracked area", this latter area being a priori an unknown, finds a clear meaning only in a 1-D setting. Secondly, several complications arise pertaining to the issue of mode separability for a crack in layered materials. In fact, modes may be non-separable in layered materials, in general. In the literature, one finds many growth laws that have been used for studying homogeneous materials. None has been successfully applied to the study of layered materials. 
Based on these considerations, one finds that current LEFM concepts do not necessarily deliver the proper tools to predict crack growth in layered materials. The purpose of the research that is reported herein is associated with the development of a fracture model that circumvents the use of LEFM and that which has the potential to address a wide range of crack problems of laminated composites.

Due to the presence of a crack within a structure, the structure behaves in two different ways. One is a local behavior associated with the crack tip, and the other is a global behavior which is a reaction against external loadings. The spring foundation model can separate out the local and global behavior of the structure. Once they are separated out, the global behavior can be characterized easily using current available mechanics theories such as beam, plate, shell, 2-D or 3-D elasticity theory. Some authors [4-11] previously have tried to solve crack problems using spring models. Some of the models do not lend themselves to a clear physical interpretation of what the spring foundation is associated with. The present work has improved upon the beam on spring foundation models by taking the beam thickness as a spring length and using a non-uniform strain distribution throughout the spring length. The present work also uses a constitutive law for the spring foundation that is based on the interatomic potential law.

The basic model is developed in Section 2.1 and the corresponding spring foundation is characterized in Section 2.2. Finite element implementation for the solution of the current model is illustrated in Section 3. Expcrimental details arc reported in Scction 4. The results from the current model are compared to the experimental results to validate the current model in Section 5. Finally, Section 6 presents conclusions and discussions.

\section{MODELING OF MODE I FAILURE}

\subsection{Basic model}

A DCB type specimen is used to model the mode I failure of laminated composites. Figure l(a) shows the specimen under mode I failure. The specimen is unidirectionally laminated and the crack is assumed to be propagating along the center of the resin rich layer, making the beam symmetric with respect to the beam centroidal line. This DCB specimen is approximated as a beam on a nonlinear elastic foundation, depicted in Fig. 1(b). The nonlinear elastic foundation is represented by an infinite number of 1-D springs. Only one half of the beam is considered due to the symmetry.

The DCR specimen reacts in two different ways against the external loading, as mentioned in the previous section. One is a local behavior originated from the crack tip and the other is a global

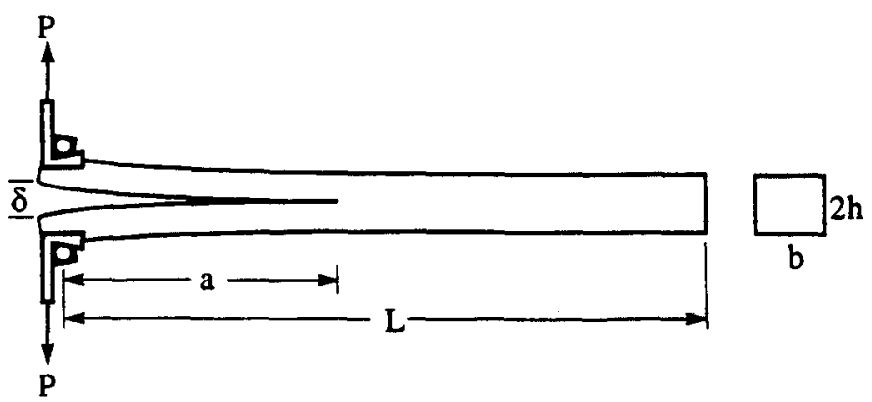

Fig. I. (a) DCB specimen under mode I failure.

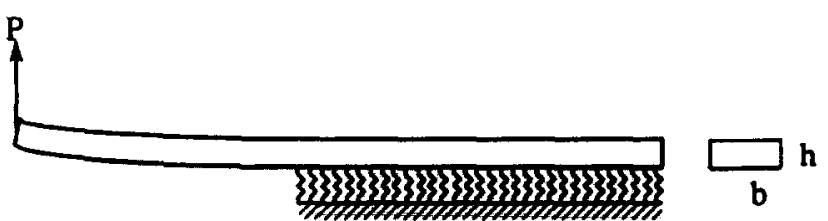

Fig. 1. (b) Beam on spring foundation model. 
behavior of the beam structure caused by the external loading. Accordingly, the DCB specimen can be split into two parts corresponding to each behavior. One is the spring foundation representative of the local behavior and the other is the beam for the global behavior. As the external loading increases, at a certain point, the first spring at the crack tip reaches its endurable maximum strength and breaks permanently, defining what is meant by crack propagation.

There are many well established theories to solve for the beam part. Euler-Bernoulli beam theory is adopted in this study for simplicity. Next, the spring foundation should be characterized to complete the current model. This is done in the following section in a systematic way. Since the microscopic behavior of the material near the crack tip is not fully known yet, some fundamental results from previous work will be used to help characterize the spring foundation properties, and a few assumptions will be made where needed.

\subsection{Characterization of the spring foundation}

There are two sources of information needed to characterize the spring foundation. One is the constitutive behavior of the material and the other is the strain-displacement relation throughout the spring length. The microscopic constitutive behavior of the material near the crack tip is somewhat different from the bulk behavior that is obtained from uniaxial tension tests performed in laboratories. Therefore, the bulk data of uniaxial tension tests may not necessarily be a proper characterization of the material near the crack tip. The material near the crack tip experiences high stress concentration. Besides, the resin rich layer which contains the crack tip is very thin and confined by two stiff plies up and down creating a constraining effect (essentially 3-D problem). Thus, it can be assumed that the material near the crack tip is more likely to behave according to the interatomic force law rather than the constitutive law obtained from a uniaxial tension test of the bulk material.

The physical meaning of fracture is that a series of two adjacent atoms are separated out permanently. Polymeric material has many kinds of bond types in it which links monomers first and then polymer chains. Some examples are carbon to carbon bonds, hydrogen bonds and Van der Waals bonds. Therefore, the fracture of polymeric materials accompanies a breaking of some combination of the different types of bonds present. No matter what bonds are broken, there should be a separation of interatomic bonds. Many kinds of interatomic potential laws have been suggested by several former researchers [12], but they all have basically the same feature that the interatomic force increases to negative infinity at compression, and it increases up to maximum strength and then decreases thereafter, approaching zero at positive infinite tension. Morse's interatomic potential law [12] is chosen in this study to represent the constitutive law of the microscopically behaving material near the crack tip. The energy of interaction $U$, between two atoms of separation $r$, is given by the Morse function as

$$
U=U_{0}\left(\mathrm{e}^{-2 a\left(r-r_{0}\right)}-2 \mathrm{e}^{-a\left(r-r_{0}\right)}\right)
$$

A slight modification of the Morse potential was made to derive a constitutive law by differentiating the potential with respect to atomic distance $r$ and replacing $a\left(r-r_{0}\right)$ with strain. The result is eq. (2) and depicted in Fig. 2.

The spring length is equal to the beam thickness in the current model. Every single spring is divided into two parts in a series. The first part is the representation of the resin rich layer which posseses the crack tip and experiences very large stresses and breaks eventually, and the second part is the representation of all the plies and resin layers above the resin layer after homogenization. The resin layer which possesses the crack tip is assumed to deform according to a nonlinear Morse function and the beam part representing all the plies above the resin layer is assumed to behave linearly. Therefore we can write down two constitutive relations for each part:

$$
\begin{gathered}
\sigma_{\mathrm{m}}=\alpha\left(\mathrm{e}^{-\beta \epsilon_{\mathrm{m}}}-\mathrm{e}^{-2 \beta c_{\mathrm{m}}}\right), \\
\sigma_{\mathrm{p}}=E_{\mathrm{p}} \epsilon_{\mathrm{p}},
\end{gathered}
$$

where $\mathrm{m}$ and $\mathrm{p}$ stand for resin layer and all the plies above the resin layer, respectively. The parameters $\alpha$ and $\beta$, in the first equation, can be determined using two conditions. The first condition is that the initial slope of eq. (2) be equal to the Young's modulus of the material, and 


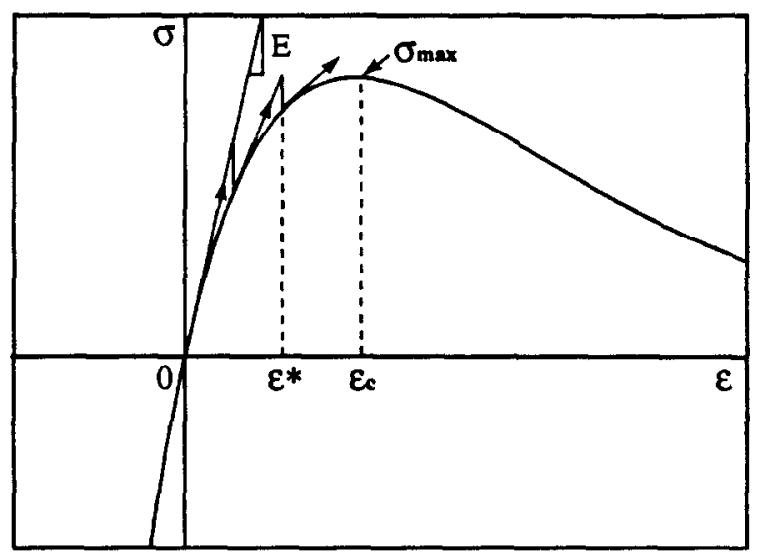

Fig. 2. Stress-strain relation based on interatomic force law and its schematic of multilinear model.

the second condition is that the maximum strength at which the slope of the constitutive equation becomes zero be equal to the ultimate tensile strength of the material. After applying these two conditions we get

$$
\begin{gathered}
\alpha=4 \sigma_{\mathrm{t}}, \\
\beta=E_{\mathrm{m}} /\left(4 \sigma_{\mathrm{t}}\right) .
\end{gathered}
$$

$\sigma_{\mathrm{t}}$ in the above equation is the material's ultimate tensile strength, and the value of this strength is different from the value observed in laboratory uniaxial tension tests for bulk material. Griffith, in his early work [13], observed the size effect on the ultimate strength of a material using glass fibers by changing the fiber diameters. He found that the strength of the material approached the theoretical fracture strength as the fiber diameter tended to zero and that it approached the bulk strength as the diameter became larger. Smook et al. [14] observed a very similar phenomenon using high polymer fibers of UHMWPE (ultra-high molecular weight polyethylene), and he proposed an equation which curve-fits his data. Chai $[2,3]$ observed the effect of resin layer thickness on the fracture toughness using adhesive joints. He found that as the resin layer thickness became very small, the fracture toughness incrcased instcad of decrcasing continuously. The reason for this volume effect on material strength can be attributed to the fact that as the volume decreases the flaw density also decreases, making the material much stronger than the bulk state.

Although the diameter effect on the material strength in a uniaxial tension test is not perfectly analogous to the thickness effect on material strength in the resin layer confined by two stiff plies up and down in the laminates, the trend of the volume effect for both cases can be very similar. Therefore, Smook's interpolation function for a polymer is employed in this study to describe the resin layer strength as a function of thickness with some modifications. The Smook interpolation function can be re-written as

$$
\sigma_{\mathrm{t}}=1 /\left(K \sqrt{D}+1 / \sigma_{0}\right)
$$

where $D$ is a fiber diameter and $\sigma_{0}$ is the strength of a flawless fiber. As the diameter becomes very large, the material strength goes to zero from the above equation. Thus, this equation is valid only up to the diameter $120 \mu \mathrm{m}$ which is the largest fiber diameter Smook used in his experiment. The resin layer thicknesses of all the specimens used in this study fall into this limit, making this equation applicable to our problem. As the diameter goes to infinity, the bulk strength of the material should be used as the value for $\sigma_{t}$. This feature has been used in the previous work [4]. Two modifications are needed to make use of the above equation in the present problem. The first one is that the resin layer thickness $2 t$ will be used instead of the diameter $D$. Secondly, a different value of $\sigma_{0}$, the ideal strength of the polymer, obtained from a perfectly ideal condition, will be used. In uniaxial tension tests of polymeric fibers, as the diameter approaches zero one single chain remains. This is the perfect condition for the calculation of the ideal strength of the polymer in 
which all the chains are fully aligned, fully packed and break simultaneously. However, this condition can never be achieved in the case of the resin layer. Even though the layer thickness approaches zero, the material failure will be still in a transverse direction ahead of the crack tip and furthermore there is still a finite width of the beam which violates the ideal condition of simultaneous breaking. Several ways of obtaining rough estimates for the ideal strength can be found. Kanninen [13] showed that for many materials the ideal strength is $E / 6$. Knott [15] showed that the ideal strength as a rough estimate is $E / 10$. Kelly and Macmillan [12] also showed several methods to estimate the ideal strength of a solid, and the results mostly fell into that range explained by Kanninen and Knott [13,15]. These values of ideal strength will be used in the above equation for $\sigma_{0}$. Figure 3 depicts the above equation for PEEK with an ideal strength of $E / 6$.

Next, the strain distribution in the spring foundation is characterized. The stress distribution near the crack tip obtained from a 2-D asymptotic solution has a $1 / \sqrt{r}$ singularity because the crack tip is assumed to be mathematically sharp. There is, of course, no such crack in reality. Taking this into account, the following equation is proposed as a modification of the 2-D asymptotic solution, where the parameter $B$ prevents the stress singularity and makes the strain distribution more realistic (Fig. 4).

$$
\begin{gathered}
\epsilon_{\mathrm{m}}=\frac{A}{\left(B^{2}+z^{2}\right)^{1 / 4}}, \quad 0 \leqslant z \leqslant t \\
\epsilon_{\mathrm{p}}=\frac{C}{z^{1 / 2}}, \quad t \leqslant z \leqslant h,
\end{gathered}
$$

where $A, B$ and $C$ are parameters to be determined. A strain distribution instead of a stress distribution is proposed because the algebra becomes impossible to carry out if a stress distribution is assumed (due to the nonlinearity). The parameter $B$ also determines the sharpness of the strain distribution throughout the resin layer thickness. The smaller $B$ is, the sharper the strain distribution becomes. As an extreme case, if $B$ approaches zero there occurs a singularity at the crack tip, and if $B$ approaches infinity the strain distribution becomes constant all throughout the resin layer thickness.

The above two equations should satisfy two conditions. The first is that the stress at the interface should be the same. Secondly, the transverse deformation of the beam is equated to the deformation of the spring foundation since there is no transverse deformation of the beam itself, i.e.

$$
\sigma_{\mathrm{m}}(z=t)=\sigma_{\mathrm{p}}(z=t)
$$

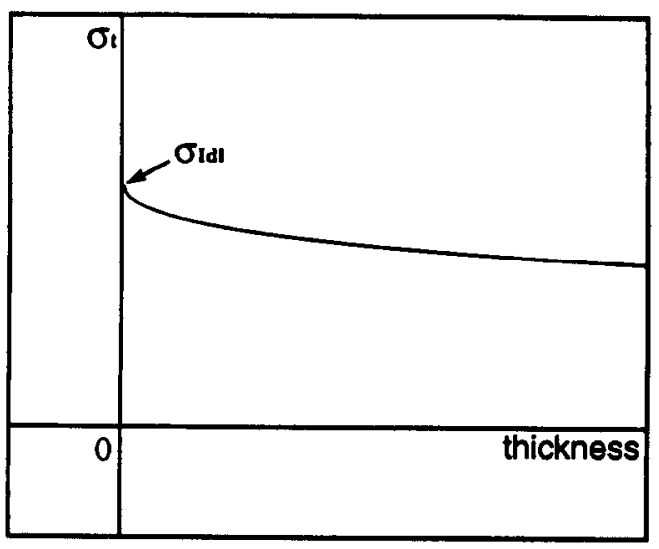

Fig. 3. Volume dependence of material strength.

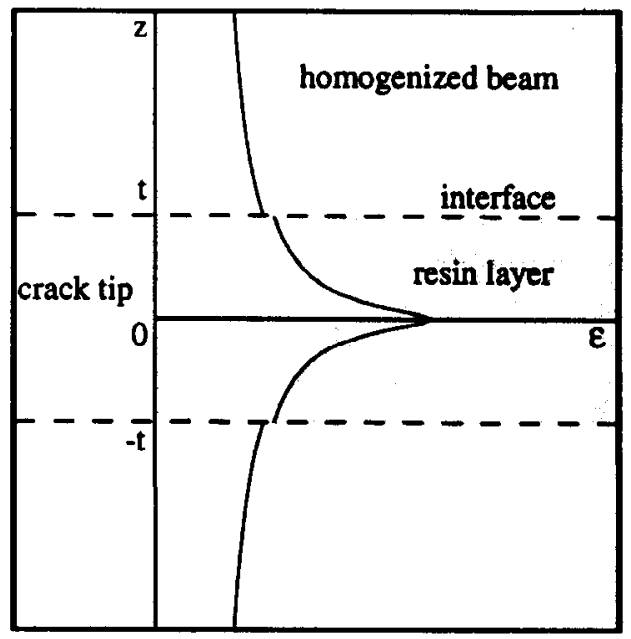

Fig. 4. Strain distribution throughout the spring length. 


$$
w=\int_{0}^{t} \epsilon_{\mathrm{m}} \mathrm{d} z+\int_{t}^{h} \epsilon_{\mathrm{p}} \mathrm{d} z .
$$

After performing the algebra, the parameters $A$ and $C$ are determined as a function of $w$. Thus the eqs (7) and (8) can be rewritten as

$$
\begin{gathered}
\epsilon_{\mathrm{m}}=\frac{w}{\psi\left(B^{2}+z^{2}\right)^{1 / 4}}, \\
\epsilon_{\mathrm{p}}=\frac{\phi w}{\psi \sqrt{z}},
\end{gathered}
$$

where $\phi$ and $\psi$ are given by

$$
\begin{gathered}
\phi=\left(\frac{E_{\mathrm{m}}}{E_{\mathrm{p}}}\right) \frac{\sqrt{t}}{\left(B^{2}+t^{2}\right)^{1 / 4}} \\
\psi=\int_{0}^{t} \frac{\mathrm{d} z}{\left(B^{2}+z^{2}\right)^{1 / 4}}+2 \phi(\sqrt{h}-\sqrt{t}) .
\end{gathered}
$$

Wang [16] investigated the crack tip stress field in a DCB adhesive specimen using a finite element analysis. He showed that the slope of the strain distribution away from the crack tip depends on both the adhesive layer thickness and the ratio of Young's modulus of the adherent to that of the adhesive. Near the crack tip, the slopes are independent of the thickness and the ratio of Young's moduli due to the stress singularity. Thus we can assume $B$ as a function of the resin layer thickness and the ratio of the Young's modulus,

$$
B=\xi(2 t)^{4}\left(\frac{E_{\mathrm{p}}}{E_{\mathrm{ut}}}\right)^{r} .
$$

In this equation, $\xi, q$ and $r$ are constants. $q$ and $r$ have been determined to be -0.5 and 0.2 , respectively by matching the slopes of Wang's results near the interface to eq. (11). $\xi$ is still an unknown constant which will be determined later.

\section{FINITE ELEMENT IMPLEMENTATION}

The finite element method was employed to solve the beam on a nonlinear elastic foundation model. A finite element code was written for this purpose, which can incorporate material nonlinearities. In the following paragraphs, a brief explanation is given of how the element matrix equation was derived using the potential energy theorem.

A multi-linear constitutive law model, depicted in Fig. 2, was used to model the material nonlinearity of the spring foundation. A nonlinear spring has in general a 1-D constitutive law as

$$
\sigma=g(\epsilon)
$$

If we suppose the strain of the spring foundation is currently at $\epsilon^{*}$ in an incremental way (Fig. 2), a linear constitutive law at the next increment can be written as

$$
\sigma=E_{1} \epsilon+E_{2},
$$

where $E_{1}$ and $E_{2}$ can be calculated as

$$
\begin{gathered}
E_{1}=g^{\prime}\left(c^{*}\right), \\
E_{2}=g\left(\epsilon^{*}\right)-\epsilon^{*} g^{\prime}\left(\epsilon^{*}\right) .
\end{gathered}
$$

For the present problem, $g(\epsilon)$ will be replaced by eq. (2). Now the potential energy for the linearized system can be written as

$$
\Pi=U_{\text {Beam }}+U_{\text {Spring }}-W_{\mathrm{e}},
$$


Table 1. Dimensions and material properties of the DCB specimens

\begin{tabular}{cccccccccc}
\hline Specimen & $\begin{array}{c}L \\
(\mathrm{~mm})\end{array}$ & $\begin{array}{c}b \\
(\mathrm{~mm})\end{array}$ & $\begin{array}{c}2 h \\
(\mathrm{~mm})\end{array}$ & $\begin{array}{c}2 t \\
(\mathrm{~mm})\end{array}$ & $\begin{array}{c}E_{\mathrm{m}} \\
(\mathrm{Gpa})\end{array}$ & $\begin{array}{c}E_{\mathrm{f}} \\
(\mathrm{Gpa})\end{array}$ & $\begin{array}{c}E_{\mathrm{b}}^{*} \\
(\mathrm{Gpa})\end{array}$ & $\begin{array}{c}\text { Frac. energy } \\
(\mathrm{N} / \mathrm{m})\end{array}$ \\
\hline E7T1/G40 & 200 & 15.5 & 9.3 & 35 & 4.1 & 286.8 & 116 & 335 \\
E719/IM7 & 200 & 15.1 & 6.45 & 6 & 3.3 & 304.1 & 135 & 1130 \\
\hline
\end{tabular}

*Shear factor is included.

where $U$ denotes the strain energy and $W_{\mathrm{c}}$ is the external work potential. Each of them are as follows;

$$
\begin{gathered}
U_{\text {Beam }}=\frac{1}{2} E I \int_{0}^{L}\left(w^{\prime \prime}\right)^{2} \mathrm{~d} x, \\
U_{\text {Spring }}=b \int_{0}^{L} \int_{0}^{t} \int_{c_{\mathrm{m}}^{*}}^{t_{\mathrm{m}}} \sigma_{\mathrm{m}} \mathrm{d} \epsilon_{\mathrm{m}} \mathrm{d} z \mathrm{~d} x+b \int_{0}^{L} \int_{1}^{h} \int_{0}^{t_{\mathrm{p}}} \sigma_{\mathrm{p}} \mathrm{d} \epsilon_{\mathrm{p}} \mathrm{d} z \mathrm{~d} x+U^{*}, \\
W_{\mathrm{e}}=w_{1} F_{1}+\theta_{1} M_{1}+w_{2} F_{2}+\theta_{2} M_{2},
\end{gathered}
$$

where $U^{*}$ is the strain energy the resin layer has absorbed up to $\epsilon^{*}$. Now the deflection $w$ can be approximated using bcam shape functions.

$$
w(x)=w_{1} N_{1}+\theta_{1} N_{2}+w_{2} N_{3}+\theta_{2} N_{4},
$$

where $N_{1}-N_{4}$ are Euler-Bernoulli beam shape functions. After substituting the approximated deflection $w(x)$ into eqs (21) and (22), the stationary potential energy theorem is applied to obtain an element matrix equation by differentiating the resulting energy equation with respect to nodal displacements, $w_{1}-\theta_{2}$. Each component of the element matrix equation is as follows;

$$
\begin{aligned}
K_{i j}^{\text {Beam }} & =E I \int_{0}^{L} N_{i}^{\prime \prime} N_{j}^{\prime \prime} \mathrm{d} x, \\
K_{i j}^{\text {Spring }} & =2 b \$_{1} \int_{0}^{L} N_{i} N_{j} \mathrm{~d} x, \\
R_{i} & =b \$_{2} \int_{0}^{L} N_{i} \mathrm{~d} x,
\end{aligned}
$$

where the indices $i, j$ range from 1 to $4 . R_{i}$ are the equivalent nodal loads created from the nonlinearity of the spring foundation. $\$_{1}$ and $\$_{2}$ are as follows;

$$
\begin{gathered}
\$_{1}=\int_{0}^{t} \frac{E_{1}}{2 \psi^{2}\left(B^{2}+z^{2}\right)^{1,2}} \mathrm{~d} z+\frac{E_{\mathrm{p}} \phi^{2}}{2 \psi^{2}}(\log (h)-\log (t)), \\
\$_{2}=\int_{0}^{t} \frac{E_{2}}{\psi\left(B^{2}+z^{2}\right)^{1 / 4}} \mathrm{~d} z .
\end{gathered}
$$

While deriving eqs (25)-(27) from eqs (21)-(23), $w^{*}$ was taken to be a constant to save computation time, however, with a much finer mesh [4].

A mesh was generated in such a manner that the element numbering is dense near the crack tip and becomes sparse gradually with distance from the crack tip. The resulting global equation was numbered diagonally to save computer memory and computation time, and was solved using a banded matrix solver. The numerical loading was done by incrementing the crack opening displacement at the end of the beam. The loading stopped when the first spring at the crack tip had reached the endurable maximum strength. At this stage, the program calculates how much the crack has propagated and re-meshes according to the new crack length. After reaching the final crack length in this manner where the loading switched to unloading in the experiment, the numerical loading also switched to unloading making a loading and unloading curve at the very end. The smallest mesh size at the crack tip was about $10 \mu \mathrm{m}$ and the increment of displacement was $1 \mu \mathrm{m}$. The computation time taken for one loop of loading and unloading was approximately $1-2 \mathrm{~h}$ by a NeXT workstation computer. 


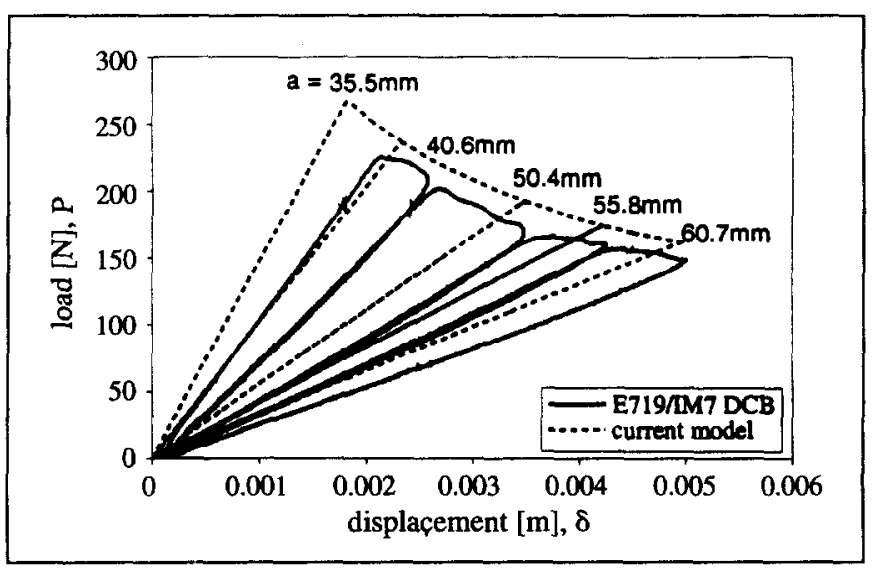

Fig. 5. Load vs displacement curves for E719/IM7 DCB specimen.

\section{EXPERIMENTS}

Mode I fracture tests were performed to validate our current spring model (Figs 5 and 6). The DCB type specimens were prepared by cutting E719/IM7 and E7T1/G40 unidirectional 48 ply laminated composite plates obtained from BP Chemical Corp. The dimensions and material properties of each specimen are shown in Table 1 . The grips were specially designed to minimize any unnecessary moment and shear arising from the loading (Fig. 1(a)). The ioading was done slowly at a speed of $0.4 \mathrm{~mm} / \mathrm{min}$ on a screw driven Riehele testing frame, simulating static conditions. The crack opening displacement at the DCB ends was measured via an LVDT and the corresponding load was measured via a $200 \mathrm{lbf}$ load cell. The instantaneous location of the crack tip was marked on one side of the beam which was pre-painted white to help read the crack tip more accurately with the aid of a magnifying glass. Both the data and time were acquired via an in-house data acquisition system. Figures 5 and 6 show the results of pulling force vs crack opening displacement curves for both specimens.

After the tests, the sides of each cracked specimen were examined using a microscope along the crack line. It was found that the failure was fully interlaminar (not intralaminar) and cohesive even though the crack did not always propagate along the center of the resin layer. The thickness of the resin layer for each specimen was measured by taking the average of several measurements along the resin layer possessing the crack. The resin layer thickness of the E719/IM7 specimen was found to be $6 \mu \mathrm{m}$ and that of the E7T1/G40 specimen was $35 \mu \mathrm{m}$. Fracture toughnesses of each specimen were calculated using the energy method [17] by dividing the area of the loading and unloading cycles by the area of the cracked surface. Three point bending tests were done to obtain

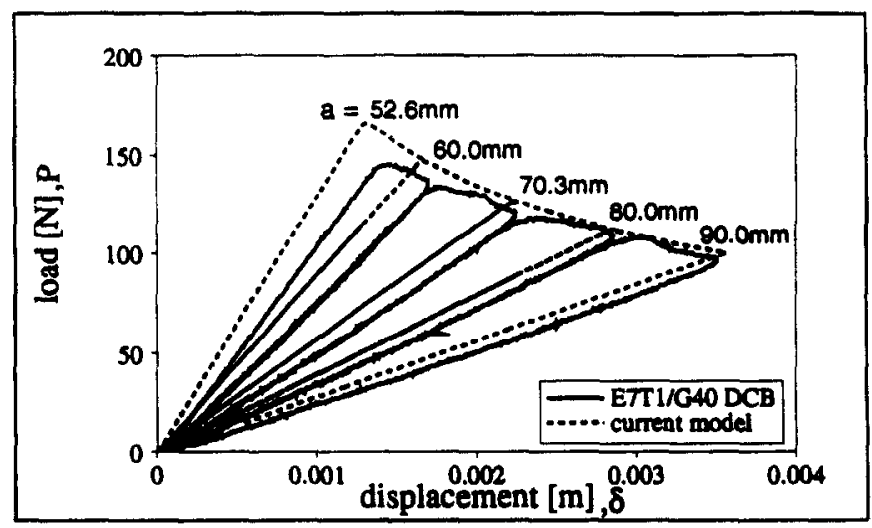

Fig. 6. Load vs displacement curves for E7T1/G40 DCB specimen. 


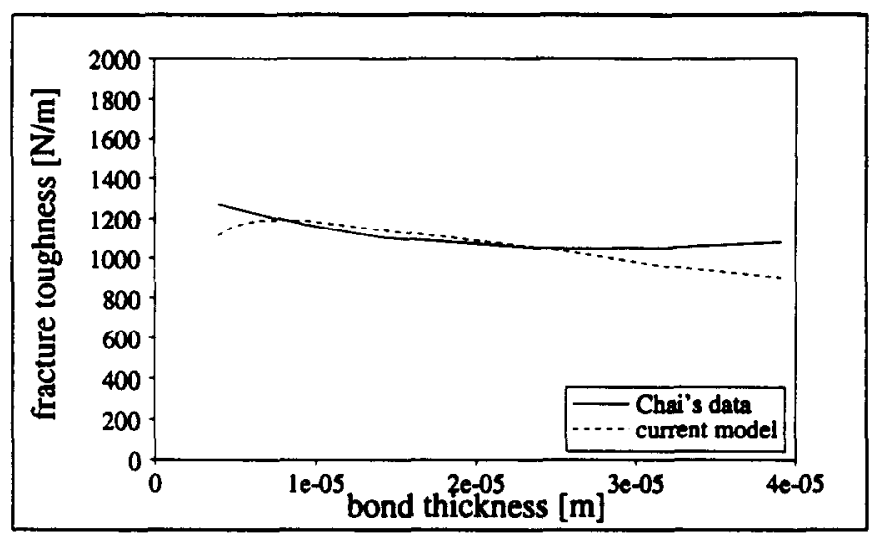

Fig. 7. Comparisons between Chai's data and current model for PEEK adhesive joint.

the elastic moduli in the beam direction of each beam. The elastic modulus in the transverse direction was calculated using Halpin and Tsai's experimental formula [18].

Chai's data $[2,3]$ as well as experimental data obtained by the authors was used in the development and verification of the current model. Chai performed a series of mode I fracture tests using adhesive joints to examine the thickness effect on fracture toughness. He used aluminum beams as adherend and PEEK (thermoplastic) and BP907 (toughened thermoset) resins as adhesives. The failure was all cohesive for these resins, thus making his data applicable to the current model. Chai's $[2,3]$ data are reproduced in Figs 7 and 8 for the thickness range in which crack propagation was stable. The current model showed only stable crack growth. Thus, only the thickness range for which the crack growth was stable was compared to the present model predictions.

\section{RESULTS}

The current model simulated several experiments; Chai's adhesive joints and mode I tests of the authors' laboratory specimens. First, the current model was applied to Chai's PEEK adhesive joints to determine the value of $\xi$ which was an unknown in Section 2 . The unknown constant $\xi$ was chosen to be $1.3 \times 10^{-9}$ by using a trial and error method until the simulation output matched Chai's data most closely. Figure 7 shows the comparisons between Chai's experimental data of PEEK adhesive joint and the output of the current model. E/6 was used as an ideal strength. The current model reproduced Chai's data very closely for a wide range of adhesive thickness. Once the value for $\xi$ is obtained, this value was retained as a constant for any material.

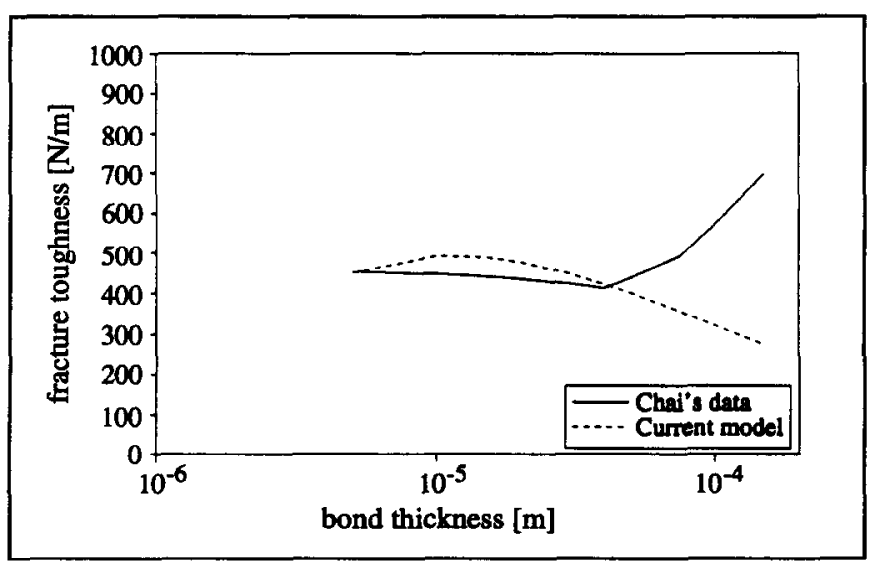

Fig. 8. Comparisons between Chai's data and current model for BP907 adhesive joint. 
The current model was next applied to a specimen made of E719/IM7 in which the resin E719 has similar chemical properties to PEEK in that they both have linear or near linear chains. The simulation result is shown in Fig. 5. The same formula of $E / 6$ was used as an ideal strength and the same value of $\xi$ was used as in Chai's PEEK adhesive joint. The result shows an excellent agreement with the experimental data of loading vs crack opening displacement curves for crack lengths where the beam theory becomes more valid, which is toward the last loading and unloading curves. The fracture toughness from the experiment is $1130 \mathrm{~J} / \mathrm{m}^{2}$, and that from our simulation is $1225 \mathrm{~J} / \mathrm{m}^{2}$.

Next, the current model was applied to Chai's BP907 adhesive joints. The resin BP907 is a toughened epoxy, so it has a different chemical structure from thermoplastic. Due to the nature of cross links, the ideal strength of a thermoset is expected to be somewhat lower than that of a thermoplastic. At the final stage of material failure, the thermoset material does not form a chain alignment, whereas thermoplastics form such an alignment producing a breaking of strong $\mathrm{C}-\mathrm{C}$ bonds. Thus a slightly lower value of ideal strength of $E / 8$ was used for this resin (BP907). The same value of $\xi$ as before was also used here. Figure 8 shows the results of a simulation output. The results agree with experiment very closely for a wide range of practical adhesive thickness.

Finally, the current model was applied to the E7T1/G40 specimen. E7T1 is a regular thermoset. Thus an even lower ideal strength than BP907 was used. Figure 6 shows the simulation results for this specimen after substituting an ideal strength of $E / 10$ and the same value of $\xi$ as before into the current model. The result is in excellent agreement with experiment in the region where beam theory becomes more valid. The fracture toughnesses calculated from experiment and simulation are $335 \mathrm{~J} / \mathrm{m}^{2}$ and $360 \mathrm{~J} / \mathrm{m}^{2}$, respectively.

\section{CONCLUDING REMARKS}

The mechanical model to simulate mode I failure in a unidirectional polymeric composite DCB type specimen reproduced Chai's experimental data very closely for a wide range of practical resin layer thickness. It also matched laboratory fracture test results obtained by the authors very closely for large crack lengths for which beam theory becomes more valid. The use of a better beam theory would result in better predictions for shorter crack lengths.

The strength of the current model is that a global behavior of the beam created from external loading and a local behavior due to the presence of the crack tip are separated out. We already possess well established mechanics theories for modeling global behavior of structures (beams, plate, shell, 2-D and 3-D elasticity theories, etc.). Once the spring foundation is well characterized, the delamination problem of composite structures can be readily analyzed utilizing the present model.

The axial and shear energy near the crack tip were not explicitly taken into account in this model, but they were included while characterizing the spring foundation through the parameter $\xi$.

If a mode II spring foundation is added to the current mode I spring foundation model, mixed mode problems can be solved without having to consider mode mixity. This new model can easily be used to predict the evolution of any shape of 2-D crack geometry. Indeed, the instantaneous planform shape of a growing crack is an output of the current model in a 2-D setting. Thus, with the use of the current model, "where" and "how much" the crack propagates by will be a part of the solution that is obtained.

Note added in proof. Subsequent to the work reported here, Timoshenko beam theory has been employed to model the crack flanges, and the agreement between theory and experiment has improved considerably. Figures 5 and 6 with Timoshenko theory are shown in ref. [19].

Acknowledgements - During the course of this work, the second author received partial funding from the ONR, Mechanics Division. We are thankful for this support. The interest and help of Dr Y. D. S. Rajapakse from the ONR, Mechanics Division is gratefully acknowledged. The beam samples used in the study were donated by BP Chemicals Corp.. CA. We would like to thank Drs E. Babayan and A. Bosch for their support. 


\section{REFERENCES}

[1] J. W. Hutchinson and Z. Suo, Mixed mode cracking in layered materials. Adv. appl. Mech. Vol 28 (1991).

[2] H. Chai, On the correlation between the Mode I failure of adhesive joints and laminated composites. Engng Fracture Mech. 24(3), 413-431 (1986).

[3] H. Chai, Bond thickness effect in adhesive joints and its significance for Mode I interlaminar fracture of composites. Proc. 7 th Conf. on Composite Materials. ASTM (1986).

[4] S. Song and A. Waas, A nonlinear elastic foundation model for interlaminar fracture of laminated composites. Composites Engng 3(10), 945-959 (1993).

[5] R. Hoff, C. A. Rubin and G. T. Hahn, A new finite element technique for modelling stable crack growth. Engng Fracture Mech. 23(1), 105-118 (1986).

[6] M. F. Kanninen, An augmented double cantilever beam model for studying crack propagation and arrest. Int. J. Fracture 9(1) (1973).

[7] M. F. Kanninen, A dynamic analysis of unstable crack propagation and arrest in the DCB test specimen. Int. J. Fracture 10(3) (1974).

[8] A. Leonardi, F. Maceri and E. Sacco, Debonding of bimodular plates. Int. Series numer. Math. 101, 113-128 (1991).

[9] P. E. Roelfstra, Simulation of failure in computer generated structures. Proc. Fracture Toughness and Fracture Energy (pp. 313-324). Sendai (12-14 October 1988).

[10] D. Shilkrut, Unilateral contact between a geometrically non-linear plate and elastic foundation. Proc. 2nd Meeting on Unilateral Problems in Structural Analysis. (17-20 June 1985).

[11] T. Ungsuwarungsri and W. G. Knauss, The role of damage-softened material behavior in the fracture of composites and adhesives. Int. J. Fracture 35, 221-241 (1987).

[12] A. Kelly and N. H. Macmillan, Strong Solids, 3rd Edition. Oxford Science Publications, Oxford (1986).

[13] M. F. Kanninen, Advanced Fracture Mechanics. Oxford Engineering Science Series, No. 15 (1985).

[14] J. Smook, W. Hamersma and W. Pennings, The fracture process of ultra-high strength polyethylene fibers. J. mater. Sci. 19, 1359-1373 (1984).

[15] J. F. Knott, Fundamentals of Fracture Mechanics. Butterworth, London (1973).

[16] S. S. Wang, An analysis of the crack tip stress field in DCB adhesive fracture specimens. Int. J. Fracture 14(1) (1978).

[17] J. G. Willians, S. Hashemi and A. J. Kinloch, The analysis of interlaminar fracture in uniaxial fibre-polymer composites. Proc. R. Soc. Lond. A427, 173-199 (1990).

[18] J. C. Halpin and S. W. Tsai, Effects of environmental factors on composite materials. AFML-TR 67-423 (June 1969).

[19] S. I. Song, A new approach to fracture of layered fibrous composites. Ph.D thesis, University of Michigan. Dept of Acrospace Engineering (April 1994). 